\title{
Transcriptional and Posttranscriptional Regulation of the CTNS Gene
}

\author{
SERENA CORALLINI, ANNA TARANTA, FRANCESCO BELLOMO, ALESSIA PALMA, ANNA PASTORE, \\ AND FRANCESCO EMMA
}

\author{
Division of Nephrology and Dialysis [S.C., A.T., F.B., A. Pal., F.E.], Laboratory of Biochemistry [A. Pas.], Bambino Gesù Children's \\ Hospital and Research Institute, 00165 Rome, Italy
}

\begin{abstract}
Cell cysteine (Cys) levels and/or the [Cys/CySS] redox potential have been shown to regulate mRNA levels of the CTNS gene, which encodes for a lysosomal cystine (CySS) carrier that is defective in cystinosis. To investigate the mechanisms involved CTNS mRNA regulation, different portions of the CTNS promotor were cloned into a luciferase vector and transfected in HK2 cells. A 1.5-2.4-fold increase in luciferase activity was observed when cells were incubated in culture medium containing low CySS concentrations. Conversely, CTNS mRNA levels decreased by $47-$ $56 \%$ in the presence of $N$-acetyl-L-cysteine (NAC). Chase experiments with actinomycin D (ActD) demonstrated a 3-fold stabilization of the CTNS mRNA when cells were cultured in low CySS medium for $48 \mathrm{~h}$. Treatment of control cells with cyclohexamide (CHX) increased CTNS mRNA levels, suggesting that CHX blocked the synthesis of proteins involved in mRNA degradation or in repression of the CTNS gene. Finally, in vitro binding assays showed increased binding $(30-110 \%)$ of the Sp-1 transcription factor to two regions of the CTNS promotor when cells were incubated in low CySS medium. These results indicate that the CTNS gene is actively regulated at the transcriptional and posttranscriptional levels and suggest that CTNS plays a pivotal role in regulating cell thiol concentrations. (Pediatr Res 70: 130-135, 2011)
\end{abstract}

$\mathrm{C}$ ystinosis is a rare autosomal recessive disease caused by mutations in CTNS gene, which encodes for cystinosin (1). In its most severe form, termed nephropathic cystinosis (NC), the disease is characterized by early onset renal Fanconi syndrome leading to renal failure and by corneal cystine (CySS) crystal depositions. Thereafter, other symptoms develop as CySS accumulates in tissues (1) (2).

The CTNS gene encodes for a CySS carrier that is primarily localized in the lysosomal membrane (3). A second isoform, termed cystinosin-LKG, originates from a differential splicing of the last exon and has been shown to encode for a protein that is targeted also to other cell compartments (4). Mutations in the CTNS gene are detected in the majority of patients with NC (1,5). In addition, mutations in the CTNS promotor have been found in three patients (6). In other cell models, cell concentration of amino acids have been shown to modulate gene activity and expression $(7,8)$. For example, amino acid

Received November 4, 2010; accepted February 4, 2011.

Correspondence: Francesco Emma, M.D., Division of Nephrology and Dialysis, Department of Nephrology and Urology, Ospedale Bambino Gesù-IRCCS, Piazza S. Onofrio 4, 00165 Rome, Italy; e-mail: francesco.emma@ opbg.net

Supported by a grant from Cystinosis Research Foundation and in part by a grant from the Cystinosis Research Network. deprivation stimulates the expression of transporters such as the system A subtype, neutral amino acid transporter A2, CD98 light chain, cationic amino acid transporter-1, or the cystine/glutamate transporter (9-12). Transcriptional regulation of these genes is often mediated by specific sequences, termed amino acid responsive elements (AAREs), that are located in the promotor regions and, occasionally, in the first intronic regions (13).

Recently, we have reported that CTNS gene expression is regulated by intracellular thiols in HK2 human kidney cells. CySS and cysteine (Cys) depletion for $48 \mathrm{~h}$ caused a 2-folds increase in CTNS mRNA that paralleled with an increase in the expression of cystinosin (14). Changes in CTNS transcript levels were associated with Cys concentrations and with the redox status of the [Cys/CySS] couple. These effects were partially reverted by treatment with $\mathrm{N}$-acetyl-L-cysteine (NAC) or with glutathione ethyl ester (14). The aim of this study was to further elucidate the mechanisms that regulate CTNS gene expression.

\section{METHODS}

Cell culture. Human kidney tubular cells (HK2, ATTCC CRL-2190) were cultured in $95 \%$ medium supplemented with 5\% FCS (Invitrogen Life Technologies, Milan, IT), ITS Liquid Media Supplement (Sigma Chemical Co.Aldrich, Milan, IT), and penicillin/streptomycin (Euro Clone, Pavia, IT). Media used in the experiments included regular DMEM containing $200 \mu \mathrm{M}$ of CySS and a "low CySS DMEM" containing $20 \mu \mathrm{M}$ CySS. This later medium was prepared by mixing in a 9:1 ratio DMEM without CySS, Cys, and methionine (Sigma Chemical Co.-Aldrich) and regular DMEM.

Construction of promotor fragments. Overlapping segments of the CTNS promotor (6) were amplified by PCR from human genomic DNA using pairs of primers listed in Table 1 and cloned in the PGL4.17 Firefly Luciferase reporter vector (Promega, Milan, IT). The Homo Sapiens clone RP11-48B14 (GenBank: AC132942.16) was used as reference with the initiation of transcription site located at position 1495 (Fig. 1).

Most amplicons encompass sequences located in the 5' UTR region. In addition, a 4862-bp fragment, spanning the entire promotor through intron 2 was cloned. This fragment contains a consensus AARE sequence near the $3^{\prime}$ end at position +3329 bp (Fig. 1). A second construct containing the promotor region and the last $400 \mathrm{bp}$ of intron 2 was also engineered. PCRs and insert cloning were performed using standard molecular biology protocols.

Transfections and luciferase detection. HK2 cells were cultured in DMEM, low CySS DMEM, or in DMEM containing $10 \mathrm{mM} \mathrm{NAC}$ for $3 \mathrm{~d}$ and starved in serum-free medium for $24 \mathrm{~h}$ before the experiments. Cells were then transiently cotransfected at $80-90 \%$ confluence with the above described

Abbreviations: AARE, amino acid responsive element; Act D, actinomycin D; CHX, cyclohexamide; Cys, cysteine; CySS, cystine; $\boldsymbol{E}_{\mathbf{h}}$, redox potential; GSH, reduced glutathione; GSSG, oxidized glutathione; NAC, $N$-acetylL-cysteine 
Table 1. Primer and oligonucleotide sequences

\begin{tabular}{|c|c|c|c|}
\hline PGL4 construct & Forward primer & Backward primer & bp \\
\hline$[-769 /-1]$ & gaccggtaccagacttcattgcgggaagggc & aacgctcgagggtcaggtgacagcggacc & 769 \\
\hline$[-348 /-1]$ & gaccggtaccaggcgagcttccacgcaacet & aacgctcgagggtcaggtgacagcggacc & 348 \\
\hline$[-316 /-1]$ & gaccggtacccggctataggeggagaggcg & aacgctcgagggtcaggtgacagcggacc & 316 \\
\hline$[-283 /-1]$ & gaccggtaccetaaagggggecсеgeccсac & aacgctcgagggtcaggtgacagcggacc & 283 \\
\hline$[-81 /-1]$ & gaccggtaccgcggaaactacaactcccag & aacgctcgagggtcaggtgacagcggacc & 81 \\
\hline$[-777 /-82]$ & gactggtaccatagagaacttcattgcgg & atatctcgagcgtcttagacggacagag & 696 \\
\hline$[-1158 /-1]$ & gaccggtacctctgtggaatgagccaggag & aacgctcgagggtcaggtgacagcggacc & 1158 \\
\hline$[-1158 /+3705]$ & gaccggtacctctgtggaatgagccaggag & gatcctcgagtctcgatttctcagaactagg & 4862 \\
\hline \multicolumn{3}{|c|}{ Oligonucleotide sequences for $\mathrm{Sp}-1$ binding assays } & Position \\
\hline (+) Strand & \multicolumn{2}{|c|}{ aggcggagaggcggcggaaggcggggttacataacttacggtaaatggccc } & {$[-308 /-284]$} \\
\hline (-) Strand & \multicolumn{2}{|c|}{ gggccatttaccgtaagttatgtaacccegecttccgecgectctccgect } & {$[-284 /-308]$} \\
\hline$(+)$ Strand & \multicolumn{2}{|c|}{ gcccaggggggcggggcaggggcggggagtcgecgttacataacttacggtaaatggccc } & {$[-440 /-407]$} \\
\hline (-) Strand & \multicolumn{2}{|c|}{ gggccatttaccgtaagttatgtaacggegactcccegcecetgccecgeccecctgggc } & {$[-407 /-440]$} \\
\hline (+) Strand & \multicolumn{2}{|c|}{ aggaggctcctggaggcggegeggececgggttacataacttacggtaaatggccc } & {$[-712 /-683]$} \\
\hline (-) Strand & \multicolumn{2}{|c|}{ gggccatttaccgtaagttatgtaacceggggecgcgecgcetccaggagcctcct } & {$[-683 /-712]$} \\
\hline
\end{tabular}

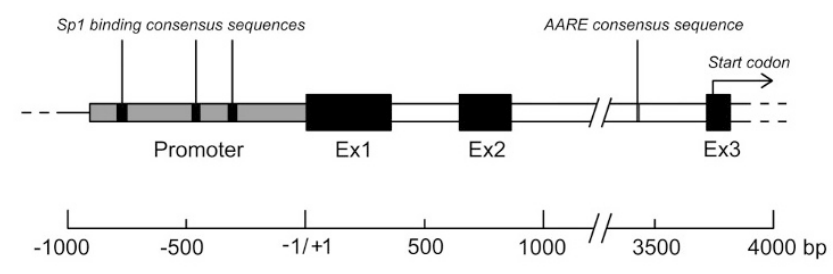

Figure 1. Schematic representation of the initial part of the CTNS gene. The promoter region is shown in gray, introns are shown in white, and exons (Ex) are shown in black. The sequences of the promoter corresponding to the oligonucleotides used for Sp-1 binding assays are highlighted in black $-308 /-284,-440 /-407$, and $-712 /-683$; the $-440 /-407$ oligonucleotide contains two $\mathrm{Sp}-1$ consensus sequences. The putative ( $5^{\prime}$-TGATGCAAA- $\left.3^{\prime}\right)$ AARE in the intron 2 at position +3329 is also highlighted in black.

reporter vectors and with the Renilla-Luciferase vector [pGL4.74(hRluc/TK), Promega], which served as control to assess transfection efficiency using Lipofectamine 2000 (Invitrogen Life Technologies). After $24 \mathrm{~h}$, cells were seeded in 96-well culture plates (Nunc, Rochester, NY) at a density of $1.9 \times$ $10^{4}$ cells per well. At $48 \mathrm{~h}$, luciferase activity was assayed using the Dual GloTM Luciferase Assay System (Promega) according to the manufacturer instructions on a Wallac Victor (2) 1420 Multilabel Counter (Perkin Elmer, Life Sciences, Shelton, CT). Promotor activities were normalized by dividing the Firefly luciferase luminescence with the Renilla luminescence. All experiments were performed at least four times in duplicate.

CTNS mRNA stability analysis and cyclohexamide chase assay. The half-life of CTNS mRNA was estimated using the transcription inhibitor actinomycin D (Act D; Sigma Chemical Co-Aldrich) at a $5 \mu \mathrm{g} / \mathrm{mL}$ concentration. The effects of protein synthesis inhibition were assessed by measuring mRNA levels in media containing $5 \mu \mathrm{g} / \mathrm{mL}$ of the translation inhibitor cyclohexamide (CHX; Sigma Chemical Co.-Aldrich).

Total RNA was isolated with TRIzol Reagent (Invitrogen Life Technologies). Oligo-p $(\mathrm{dT})_{15}$ primed cDNA strings were obtained with the First-strand cDNA synthesis kit for RT-PCR (Roche Applied Science, Indianapolis, IN) CTNS transcripts were amplified by PCR using the following pair of primers: $5^{\prime}$-ccctgaagctcgtagagaaatg-3' (forward) and $5^{\prime}$-ggaggaacatctgcaggagg- $3^{\prime}$ (backward). Glyceraldehyde 3-phosphate dehydrogenase was used as a control housekeeping gene and was amplified using the following pair of primers: $5^{\prime}$-ctgcaccaccaactgcttag-3' (forward); 5' -aggtccaccactgacacgtt-3' (backward). PCR amplicons were resolved in a $1.2 \%$-agarose gel and measured by densitometry with the Typhoon 8600 Variable Mode Imager (Amersham Pharmacia Biothec, Pittsburgh, PA) and Quantity One software (Bio-Rad Laboratories, Hercules, CA).

Sp1 binding assay. Nuclear fractions were isolated and processed using the Nuclear Extract and Sp-1 TransAM kits (Active Motif, Carlsbad, CA) that allows detection of Sp-1 binding by ELISA. Briefly, multiwell plates were coated with $10 \mu \mathrm{M}$ of custom designed biotinylated double-strand oligonucleotides, spanning the CTNS promotor regions $-308 /-284,-440 /-407$, or $-712 /-683$ (Table 1, Fig. 1). These regions contain consensus sequences for the SP1 transcription factor at positions $-299 /-290,-437 /-429,-426 /$
-418 , and $-723 /-715$. Nuclear extracts were added to coated wells and were incubated for $1 \mathrm{~h}$ at room temperature. Primary anti-Sp1 antibodies and secondary anti-rabbit-HRP-conjugated antibodies were then applied in sequence for $1 \mathrm{~h}$ at room temperature. Sp-1 binding was measured by colorimetry with the Benchmark Plus microplate spectrophotometer (Bio-Rad Laboratories) at $450 \mathrm{~nm}$, using a $655-\mathrm{nm}$ reference wavelength. Binding specificity was assayed by competition experiments, where $20 \mu \mathrm{M}$ of unlabeled double-strand oligonucleotides were added to coated wells.

Measurement of intracellular thiol levels and calculation of redox potentials. Intracellular thiols were measured by HPLC as previously reported (15). Theoretical redox potentials (16) of the GSH/GSSG and Cys/CySS couples were calculated using the Nernst equation, $E_{\mathrm{h}}=E_{0}+\mathrm{R} T / n \mathrm{~F} \ln$ ([disulfide] $/\left[\right.$ thiol] ${ }^{2}$ ), where $E_{0}$ is the standard potential for the redox couple $\left(-264 \mathrm{mV}\right.$ for $\left.\mathrm{GSSG} / \mathrm{GSH}^{2}\right),-250 \mathrm{mV}$ for $\left.\mathrm{CySS} / \mathrm{Cys}^{2}\right), \mathrm{R}$ is the gas constant, $T$ is the absolute temperature, $n$ is the number of transferred electrons (e.g. 2), and $\mathrm{F}$ is Faraday's constant (16). Calculations were made assuming a dilution volume for Cys/CySS and GSH/GSSG of $5 \mu \mathrm{L} / \mathrm{mg}$ of protein.

Statistical analysis. Statistical analyses were performed using SPSS for windows 11.0 software (SPSS Inc, Chicago, IL). All continuous data were compared with the Mann-Whitney $U$ test. All tests were two sided and considered significant for $p$ values $<0.05$.

\section{RESULTS}

CTNS mRNA changes in different cell culture conditions. As stated above, we have previously reported that CySS deprivation increases CTNS mRNA levels. Complete CySS deprivation, however, decreases substantially protein synthesis, preventing accurate measurements of luciferase activity. We have therefore conducted preliminary experiments using various concentrations of CySS in the culture medium. This allowed defining a minimum concentration of $15-20 \mu \mathrm{mol} / \mathrm{L}$ of CySS that induced maximal luciferase activity at $48 \mathrm{~h}$. At lower concentrations, cell growth and luciferase activity decreased substantially (data not shown). On these bases, experiments in "low CySS DMEM" were carried at $20 \mu \mathrm{mol} / \mathrm{L}$ of CySS (e.g. 10 times less than regular DMEM). Under these conditions, cell growth was slowed by a mean of $34 \pm 4 \%$ after $48 \mathrm{~h}(n=4)$, when cells were plated at 20-30\% confluency (data not shown). Therefore, cells cultured in low CySS medium were plated at higher density to reach a similar confluence to cell cultured in regular DMEM when luciferase activity was assessed.

CTNS mRNA levels, cell Cys, CySS, GSH, GSSG, and $E_{\mathrm{h}}$ for the [Cys/CySS] and [GSH/GSSG] redox couples under different culture conditions are reported in Table 2. As shown, CTNS mRNA increased by 2.3 -folds in cell chronically cul- 
Table 2. Changes in cell thiol levels and redox potentials in different experimental culture conditions

\begin{tabular}{|c|c|c|c|c|c|c|}
\hline Variables & Units & DMEM & Low CySS DMEM & $p$ & DMEM + NAC & $p$ \\
\hline CTNS mRNA & Relative values & $1.00 \pm 0.19$ & $2.26 \pm 0.45$ & $<0.01$ & $0.58 \pm 0.25$ & $<0.05$ \\
\hline Cys & $\mathrm{nmol} / \mathrm{mg}$ protein & $18.8 \pm 1.2$ & $7.2 \pm 0.5$ & $<0.0001$ & $76.3 \pm 3.0$ & $<0.0001$ \\
\hline CySS & $\mathrm{nmol} / \mathrm{mg}$ protein & $0.4 \pm 0.2$ & $0.4 \pm 0.1$ & NS & $2.1 \pm 0.4$ & $<0.0001$ \\
\hline$E_{\mathrm{h}}[\mathrm{Cys} / \mathrm{CySS}]$ & $\mathrm{mV}$ & $-189 \pm 35$ & $-137 \pm 16$ & $<0.05$ & $-232 \pm 25$ & $<0.05$ \\
\hline GSH & $\mathrm{nmol} / \mathrm{mg}$ protein & $195.3 \pm 12.1$ & $80.5 \pm 5.0$ & $<0.0001$ & $578.2 \pm 48.2$ & $<0.0001$ \\
\hline GSSG & $\mathrm{nmol} / \mathrm{mg}$ protein & $12.1 \pm 0.3$ & $12.4 \pm 0.3$ & NS & $17.0 \pm 1.1$ & $<0.01$ \\
\hline$E_{\mathrm{h}}[\mathrm{GSH} / \mathrm{GSSG}]$ & $\mathrm{mV}$ & $-250 \pm 17$ & $-196 \pm 12$ & $<0.01$ & $-305 \pm 23$ & $<0.01$ \\
\hline
\end{tabular}

tured in low CySS medium. In parallel, the concentration of reduced thiols decreased significantly, whereas CySS and GSSG levels remained stable; this resulted in decreased $E_{\mathrm{h}}$ for both redox couples.

Approximately the opposite effect was obtained after culturing cells in medium containing NAC. As indicated in Table 2, CTNS mRNA decreased by $42 \%$. Lower CTNS mRNA levels were associated with a significant increase in all measured thiol concentrations and $E_{\mathrm{h}}$ of both redox couples.

CySS deprivation increased CTNS promotor activity. To investigate the mechanisms underlying the regulation of CTNS mRNA levels, the promotor activity was assayed using luciferase as a reporter gene. Five overlapping fragments of the CTNS promotor (starting from $\mathrm{bp}-1$ ) were cloned into the reporter vectors (Table 1, Fig. 2A). As shown in Fig. 2A, the luciferase activity increased 1.5 to 2.4 -folds in all tested promotor segments in low CySS medium. Specifically, the luciferase activity driven by the longer promoter fragment (769 bp) increased from $331 \pm 68$ to $814 \pm 88$ Relative Luminescence Units (RLU; $p<0.001$ ), whereas cells transfected with the shorter $81 \mathrm{bp}$ fragment responded to low CySS concentration by an increase in luciferase activity from $117 \pm$ 9 to $215 \pm 18 \mathrm{RLU}(p<0.001$, Fig. $2 A)$. Longer CTNS fragments (769 bp, $348 \mathrm{bp}$, and $316 \mathrm{bp}$ ) containing the first $316 \mathrm{bp}\left(3^{\prime}\right.$ to $\left.5^{\prime}\right)$ of the promotor induced higher luciferase activity than shorter fragments $(283 \mathrm{bp}, 81 \mathrm{bp})$, indicating that the region located between bp -283 and bp -316 is critical to enhance promotor activity. All five tested promotor segments responded similarly to CySS depletion, raising the hypothesis that the first $81 \mathrm{bp}$ may contain a thiol-sensitive region. To test this hypothesis, a sixth construct encompassing CTNS sequences located between bp -81 and bp -769 was generated. As shown in Fig. $2 A$, the luciferase activity driven by this latter plasmid was similar to the activity driven by the fulllength promotor $(-1 /-769 \mathrm{bp})$.

AARE sequences are located in promotor or intronic regions of genes encoding for amino acid carriers and have been shown to regulate gene transcription in response to changes in amino acid concentrations. By electronic screening, we have identified a putative AARE sequence (5'-TGATGCAAA-3') in intron 2, near exon 3 (Fig. 1). To test whether this sequence is involved in the regulation of CTNS gene transcription, a construct spanning the promotor region to the end of intron 2 was engineered (Fig. 2B). A second construct containing the promotor region and the last $400 \mathrm{bp}$ of intron 2 was also engineered to test the effects of the putative AARE sequence when inserted closer to the promotor (Fig. 2B). The first construct elicited very low luciferase activity; the lumines-
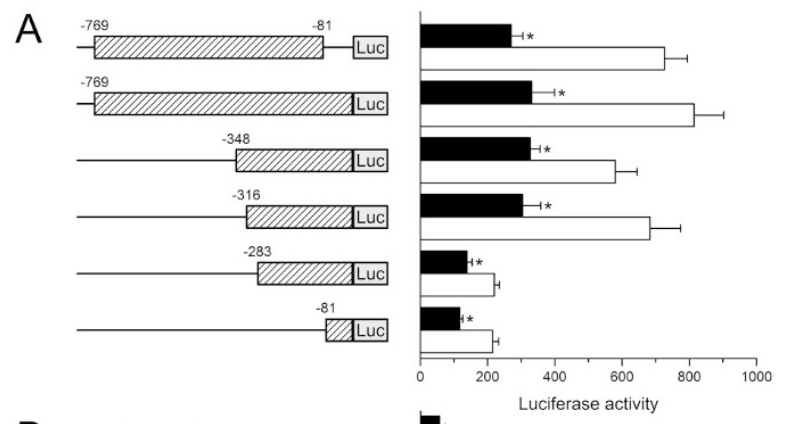

B
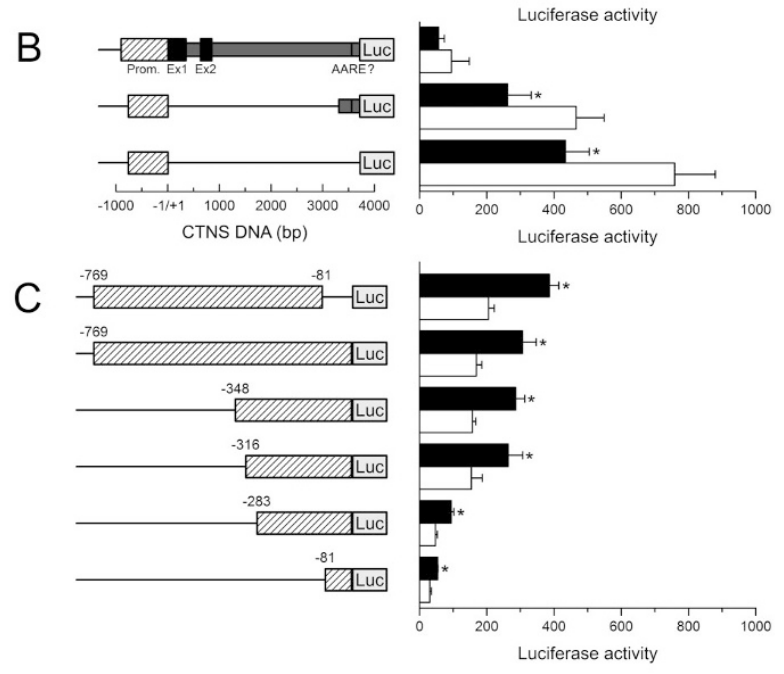

Figure 2. Luciferase activities of CTNS promotor constructs. Promotor constructs are represented on the left. Hashed bars indicate the promotor regions that are cloned into each construct. Gray bars indicate the luciferase gene (LUC). DNA coordinates are indicated in numbers and in the $x$ axis $(B)$. (A and $B$ ) Luciferase activity in control cells and cells cultured in low CySS medium $(20 \mu \mathrm{mol} / \mathrm{L})$. (C) Luciferase activity in control cells and cells cultured in medium containing $10 \mathrm{mmol} / \mathrm{L}$ of NAC. $\mathbf{\square}$ : DMEM; $\square$ : "low CySS DMEM" in $A$ and $B$ end "DMEM + NAC" in $C$; LUC $=$ luciferase. $* p<0.001$.

cence elicited by the second construct was higher but significantly lower than the luminescence elicited by plasmids containing only the full-length promotor (Fig. 2B). The AARE sequence added no noticeable sensitivity to changes in CySS concentration of the culture medium.

To confirm the CTNS promotor sensitivity to cell thiols, HK2 cells were cultured in medium containing $10 \mathrm{mM}$ NAC. These results are reported in Fig. 2C. As shown, addition of NAC to the culture medium caused a $47-56 \%$ decrease in luciferase activity. The degree of inhibition of luminescence was comparable, in relative values, for all tested constructs. In absolute values, differences between constructs paralleled those observed in low CySS medium. 

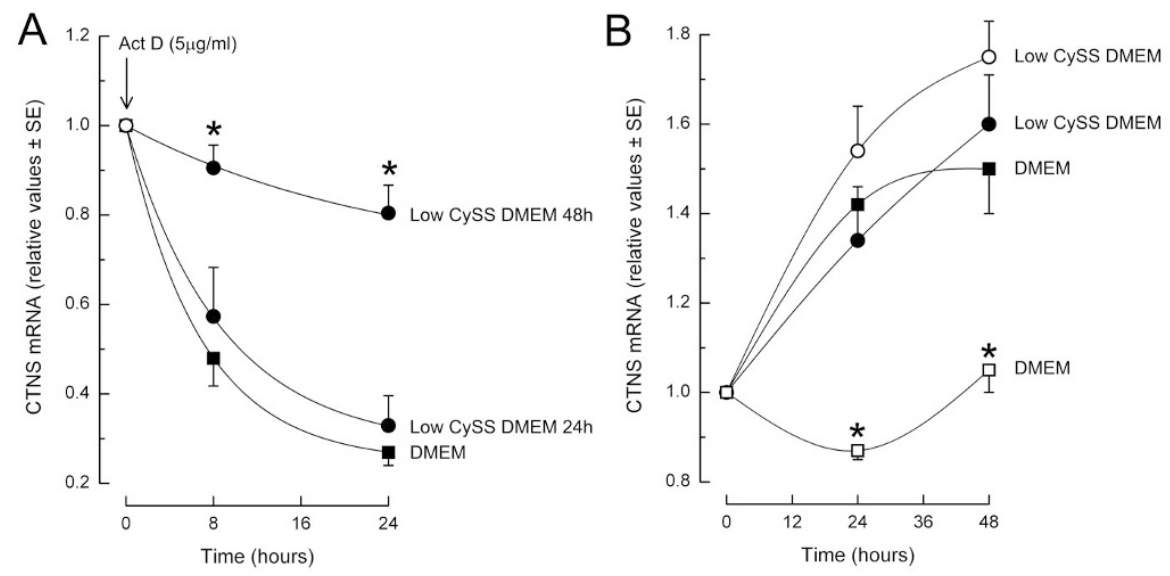

Figure 3. CTNS mRNA levels after actinomycin and CHX treatment. (A) CTNS mRNA was measured after incubating cells in the presence of Act D. Cells were maintained in regular DMEM (squares) or were switched to low CySS medium for $24 \mathrm{~h}$ or $48 \mathrm{~h}$ (circles). mRNA was measured by RT-PCR and normalized for GADPH expression. (B) CTNS mRNA was measured in the presence (filled labels) or absence (empty labels) of CHX. mRNA was measured as in $A .{ }^{*} p<0.001$.

CySS deprivation increased CTNS mRNA stability. The above data indicate that changes in CTNS mRNA levels related to CySS concentrations in the culture media are, at least in part, related to transcriptional regulation of the CTNS gene. To test whether posttranscriptional elements are also involved, cells were treated with ActD and with CHX.

As shown in Fig. 3A, CTNS mRNA levels decreased substantially from $1 \pm 0.31$ to $0.27 \pm 0.27$ (relative values) in $24 \mathrm{~h}$, after blocking transcription in cells cultured in regular DMEM. mRNA decay was similar (from $1 \pm 0.42$ to $0.33 \pm$ 0.12 in $24 \mathrm{~h}$ ) after switching cells to a low CySS medium for $24 \mathrm{~h}$ ( + duration of the chase). Conversely, if cells were switched to low CySS medium for $48 \mathrm{~h}$ before inhibiting transcription, CTNS mRNA levels decrease significantly less rapidly (from $1 \pm 0.27$ to $0.80 \pm 0.19$, Fig. $3 A$ ), indicating a substantial stabilization of the mRNA.

Translation inhibition experiments were performed after switching cells to low CySS medium in the presence or in the absence of CHX. As shown in Fig. 3B, in the absence of CHX (empty labels), mRNA levels remained stable in cells that were maintained in regular DMEM and increase after $48 \mathrm{~h}$ by $1.75 \pm 0.20$-folds $(p<0.001)$ when cell were switched to low CySS medium, as expected. In the presence of CHX, mRNA levels increased in a similar way in the latter group of cells. However, CTNS mRNA increased to levels comparable with low CySS medium in cells that were maintained in regular DMEM in the presence of CHX.

Taken together, these results indicate that CTNS mRNA degradation is an active process and raise the hypothesis that CHX blocks the synthesis of a protein or a group of proteins that are involved in this process.

CySS deprivation modifies Sp-1 binding to the CTNS promoter. The Sp-1 transcription factor has been shown to bind specifically to the CTNS promotor and mutations involving consensus sequences for $\mathrm{Sp} 1$ have been shown to cause cystinosis (6). We have tested the effects of partial CySS deprivation on Sp-1 binding in three regions of the CTNS promotor that contain four consensus sequences for $\mathrm{Sp}-1$. Nuclear fractions were collected from HK2 cells that were cultured for $5 \mathrm{~d}$ in regular DMEM or in low CySS DMEM and were assayed as described in the method section.

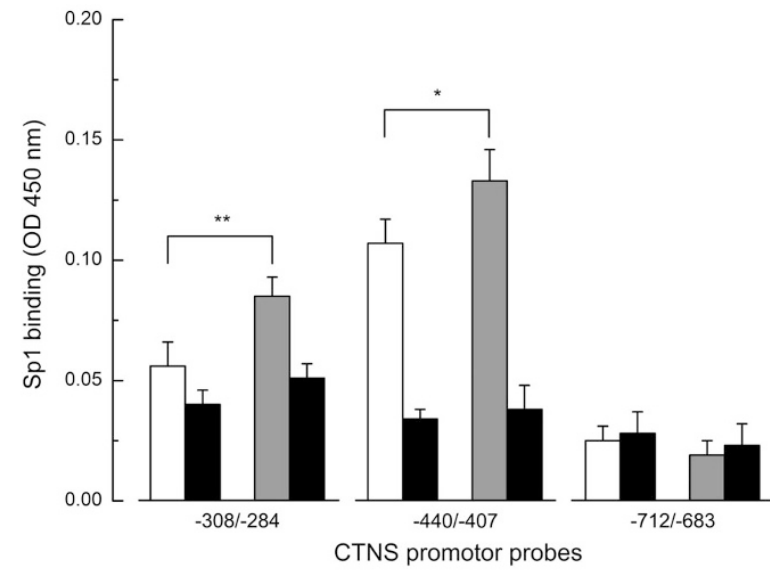

Figure 4. Sp-1 binding assay. $\mathrm{Sp} 1$ binding activities of three different CTNS promotor regions (positions $-316 /-268,-450 /-400$, and $-738 /-680$ from the translation start site). Binding of Sp-1 was assayed by ELISA (see text for details). $\square$ : DMEM; $\square$ : “low CySS DMEM." Specificity was assayed by competition assays with unlabeled competitor oligonucleotides (ם). *p $<$ $0.02, * * p<0.001$.

As shown in Fig. 4, specific binding was observed in two regions of the promotor that are included in the oligonucleotide probes $-440 /-407$ and $-308 /-284$. After partial CySS deprivation and after subtracting the nonspecific signal obtained after competition with nonlabeled oligonucleotides, Sp-1 binding increased on average by $112 \%$ using the probe $-308 /-284(p<0.001)$ and by $30 \%$ using the probe $-440 /$ $-407(p<0.02)$.

\section{DISCUSSION}

Cystinosis is a monogenic disease that is caused by mutations or deletions of CTNS gene that encodes for cystinosin $(1,3,5)$. Until recently, cystinosin was considered to be a constitutive carrier of the lysosomal membrane, allowing the efflux of CySS as it accumulates in lysosomes after protein degradation. We have recently reported that the CTNS gene also encodes for cystinosin-LKG, which is also expressed in other cell compartments, and that cell incubation in CySSdepleted medium up-regulates the expression of both CTNS isoforms $(4,14)$. By culturing cells in different conditions, 
including oxidizing conditions and after inhibiting GSH synthesis, we have shown that changes in CTNS mRNA correlate best (negative correlation) with cell Cys concentrations and with the $E_{\mathrm{h}}$ of the [Cys/CySS] redox couple (14).

To date, the physiopathology of cell damage in cystinosis has not been fully elucidated. Studies on human fibroblasts, on proximal tubular epithelial cells or using directly human tissues from patients with cystinosis, have shown that cystinotic cells are more prone to apoptosis and to autophagy, and that cysteinylation of proapoptotic kinases may be involved in this process and that in some cases mitochondrial function is impaired (17-21).

To better understand the role of cystinosin in cells, we have begun analyzing the mechanisms involved in CTNS mRNA regulation. Our results indicate that the CTNS gene is actively regulated through mechanisms that operate both at the transcriptional and posttranscriptional levels.

Other examples of gene regulation by intracellular amino acids have been reported. These may involve coordinated activation of groups of genes that encode for transcription factors, enzymes, or cell transporters in response to cell stress induced by amino acid deprivation $(7,8)$ or more targeted activation of specific transporters or enzymes. Changes in Cys concentration in HeLa cells, for example, modify the expression of several genes, including the C/EBP homologous protein transcriptional factor and the asparagine synthetase genes (22). Similarly, CySS starvation in human hepatoma cells increases the expression of genes involved in Cys metabolism and transport, in GSH synthesis and in cell growth (23). More specific gene activation often requires the interaction of enhancers with specific AARE sequences that are located in the promotor region or in the first introns of genes $(12,13,24,25)$. Disruption of the AARE sequence of the asparagine synthetase promotor, for example, inhibits gene transcription on asparagine deprivation (25). Similarly, CySS deprivation induces the transcription of the CySS/glutamate transporter through a specific AARE sequence located in its promotor (12). This same AARE sequence is present in the second intron of the CTNS gene and was therefore a cis-acting candidate region for the regulation of the CTNS gene. However, our results do not support this hypothesis. This AARE sequence of the CTNS gene may represent a remnant of an ancestral gene; alternatively, other cis- or trans-acting elements that were not active in our reporter gene system may be required to elicit its function.

Notwithstanding this result, our data clearly show that the CTNS promotor is activated when cells are cultured in low CySS medium, which causes a dramatic fall in cell thiol levels. Although these conditions are unlikely to be observed in vivo, the complex regulatory elements that we observed in vitro may play a crucial role in vivo, in particular, in the proximal tubular epithelium, which is compromised at the early stages of NC and where high amounts of CySS are reabsorbed. In addition, thiol depletion also induces cell oxidation. Although our previous data (14) support the hypothesis that high CTNS mRNA levels are driven primarily by Cys depletion rather than reactive oxygen species, we cannot rule out completely that increased CTNS mRNA are part of a broader response to oxidative stress.

The comparison of different promoter constructs activity in our study suggests that the thiol sensitivity of the CTNS promotor depends on other trans-acting elements. DNA strings encompassing $b p-283$ to $b p-316$ represent a critical region, as indicated by a marked increase in luciferase activity in transcripts containing this region and confirm the data by Phornphutkul et al. (6), who showed that a $\mathrm{G}>\mathrm{C}$ mutation at position -295 bp causes cystinosis and reduces the binding of the Sp-1 transcription factor (6), which is involved in several cell processes, including apoptosis, cell differentiation, and cell cycle (26).

On these bases, we have tested the impact of changes in medium CySS concentrations on Sp-1 binding to the CTNS promotor. In partial contrast to our gene reporter results, which showed increased promotor activity even in constructs that do not contain Sp-1 binding sites, we observed increased Sp-1 binding to the consensus sequence located at position $-308 /-283$ and to two other sequences that are contained in the $-440 /-407$ oligonucleotide (the presence of two Sp-1 consensus sequences in this region may explain higher activity in our in vitro assays). The apparent contradiction between our gene reporter data and the results of our DNA binding experiments do not exclude that both mechanisms may be involved in vivo.

Significant posttranscriptional regulation was also demonstrated. Specifically, we observed that culturing cells in low CySS medium induced a very significant stabilization of the CTNS mRNA. A thorough description of the mechanisms involved in CTNS mRNA stability was beyond the scope of the present work. Our results are reminiscent of several other biological systems, such as the "classic" model of the transferrin receptor, whose mRNA half-life depends on cell iron concentrations $(27,28)$. Mechanisms involved in mRNA stability and degradation include the action of "broad" spectrum RNAses, of gene-specific RNAses and of microRNAs, among others (29). Enzyme-mediated degradation of CTNS mRNA is in part supported by our CHX data. Hypothetically, cell culture in low CySS conditions inhibits the synthesis of a specific RNAse that decreases CTNS mRNA half-life. Alternatively, CHX could inhibit the synthesis of a repressor protein that operates at the transcriptional level.

Regardless of the underlying mechanisms, the present data demonstrate that the expression of CTNS mRNA is heavily regulated. Our previous data suggested that cytosolic Cys levels are the primary stimuli that regulate cystinosin expression (14). This would be consistent with mechanisms of regulation of other amino acid transporters. Once in the cytosol, CySS is reduced into Cys and is maintained in a reduced state by cell reducing systems, mainly by the GSH/ GSSG couple. In experimental solutions, however, Cys is readily oxidized into CySS, preventing to test the effects of different Cys concentrations on CTNS mRNA synthesis with more direct experimental models, such as nuclear run-on assays.

Similar to iron or calcium, Cys is a highly reactive compound, which is essential to a number of cell processes, 
including protein and GSH synthesis but which can also become detrimental, causing cysteinylation of proteins, when it accumulates in excess.

The present data may shed new light in understanding cell damage in cystinosis. In addition to demonstrate that CTNS mRNA levels increase when cell need to augment their thiol content, inhibition of CTNS gene expression in the presence of excess of thiols may be equally important. Hypothetically, loss of regulation of cell Cys content secondary to CTNS mutations may lead to increased cytosolic Cys content and to abnormal cysteinylation of proteins, which would make cystinotic cells more prone to apoptosis $(20,21)$. In this respect, it is interesting to notice that inhibition of CTNS mRNA by RNA interference in HK2 cells not only increases cell CySS but also cell Cys concentrations.

\section{REFERENCES}

1. Gahl WA, Thoene JG, Schneider JA Cystinosis. N Engl J Med 347:111-121, 2002

2. Theodoropoulos DS, Krasnewich D, Kaiser-Kupfer MI, Gahl WA 1993 Classic nephropathic cystinosis as an adult disease. JAMA 270:2200-2204

3. Town M, Jean G, Cherqui S, Attard M, Forestier L, Whitmore SA, Callen DF, Gribouval O, Broyer M, Bates GP, van’t Hoff W, Antignac C 1998 A novel gene encoding an integral membrane protein is mutated in nephropathic cystinosis. Nat Genet 18:319-324

4. Taranta A, Petrini S, Palma A, Mannucci L, Wilmer MJ, De Luca V, DiomediCamassei F, Corallini S, Bellomo F, van den Heuvel LP, Levtchenko EN, Emma F 2008 Identification and subcellular localization of a new cystinosin isoform. Am J Physiol Renal Physiol 294:F1101-F1108

5. Taranta A, Wilmer MJ, van den Heuvel LP, Bencivenga P, Bellomo F, Levtchenko EN, Emma F 2010 Analysis of CTNS gene transcripts in nephropathic cystinosis Pediatr Nephrol 25:1263-1267

6. Phornphutkul C, Anikster Y, Huizing M, Braun P, Brodie C, Chou JY, Gahl WA 2001 The promoter of a lysosomal membrane transporter gene, CTNS, binds Sp-1, shares sequences with the promoter of an adjacent gene, CARKL, and causes cystinosis if mutated in a critical region. Am J Hum Genet 69:712-721

7. Hyde R, Taylor PM, Hundal HS 2003 Amino acid transporters: roles in amino acid sensing and signalling in animal cells. Biochem J 373:1-18

8. Fafournoux P, Bruhat A, Jousse C 2000 Amino acid regulation of gene expression. Biochem J 351:1-12

9. Ling R, Bridges CC, Sugawara M, Fujita T, Leibach FH, Prasad PD, Ganapathy V 2001 Involvement of transporter recruitment as well as gene expression in the substrate-induced adaptive regulation of amino acid transport system A. Biochim Biophys Acta 1512:15-21

10. Campbell WA, Sah DE, Medina MM, Albina JE, Coleman WB, Thompson NL 2000 TA1/LAT-1/CD98 light chain and system L activity, but not 4F2/CD98 heavy chain, respond to arginine availability in rat hepatic cells. Loss of response in tumor cells. J Biol Chem 275:5347-5354
11. Hyatt SL, Aulak KS, Malandro M, Kilberg MS, Hatzoglou M 1997 Adaptive regulation of the cationic amino acid transporter-1 (Cat-1) in Fao cells. J Biol Chem 272:19951-19957

12. Sato H, Nomura S, Maebara K, Sato K, Tamba M, Bannai S 2004 Transcriptional control of cystine/glutamate transporter gene by amino acid deprivation. Biochem Biophys Res Commun 325:109-116

13. Palii SS, Chen H, Kilberg MS 2004 Transcriptional control of the human sodiumcoupled neutral amino acid transporter system A gene by amino acid availability is mediated by an intronic element. J Biol Chem 279:3463-3471

14. Bellomo F, Corallini S, Pastore A, Palma A, Laurenzi C, Emma F, Taranta A 2010 Modulation of CTNS gene expression by intracellular thiols. Free Radic Biol Med 48:865-872

15. Pastore A, Massoud R, Motti C, Lo Russo A, Fucci G, Cortese C, Federici G 1998 Fully automated assay for total homocysteine, cysteine, cysteinylglycine, glutathione, cysteamine, and 2-mercaptopropionylglycine in plasma and urine. Clin Chem 44:825-832

16. Jones DP, Carlson JL, Mody VC, Cai J, Lynn MJ, Sternberg P 2000 Redox state of glutathione in human plasma. Free Radic Biol Med 28:625-635

17. Levtchenko E, de Graaf-Hess A, Wilmer M, van den Heuvel L, Monnens L, Blom H 2005 Altered status of glutathione and its metabolites in cystinotic cells. Nephrol Dial Transplant 20:1828-1832

18. Mannucci L, Pastore A, Rizzo C, Piemonte F, Rizzoni G, Emma F 2006 Impaired activity of the gamma-glutamyl cycle in nephropathic cystinosis fibroblasts. Pediatr Res 59:332-335

19. Laube GF, Shah V, Stewart VC, Hargreaves IP, Haq MR, Heales SJ, van't Hoff WG 2006 Glutathione depletion and increased apoptosis rate in human cystinotic proximal tubular cells. Pediatr Nephrol 21:503-509

20. Park MA, Pejovic V, Kerisit KG, Junius S, Thoene JG 2006 Increased apoptosis in cystinotic fibroblasts and renal proximal tubule epithelial cells results from cysteinylation of protein kinase C delta. J Am Soc Nephrol 17:3167-3175

21. Sansanwal P, Yen B, Gahl WA, Ma YW, Ying LH, Wong LJ, Sarwal MM 2010 Mitochondrial autophagy promotes cellular injury in nephropathic cystinosis. J Am Soc Nephrol 21:272-283

22. Jousse C, Bruhat A, Ferrara M, Fafournoux P 2000 Evidence for multiple signaling pathways in the regulation of gene expression by amino acids in human cell lines. J Nutr 130:1555-1560

23. Lee JI, Dominy JE, Sikalidis AK, Hirschberger LL, Wang W, Stipanuk MH 2008 HepG2/C3A cells respond to cysteine deprivation by induction of the amino acid deprivation/integrated stress response pathway. Physiol Genomics 33:218-229

24. Bruhat A, Jousse C, Carraro V, Reimold AM, Ferrara M, Fafournoux P 2000 Amino acids control mammalian gene transcription: activating transcription factor 2 is essential for the amino acid responsiveness of the CHOP promoter. Mol Cell Biol 20:7192-7204

25. Guerrini L, Gong SS, Mangasarian K, Basilico C 1993 Cis- and trans-acting elements involved in amino acid regulation of asparagine synthetase gene expression. Mol Cell Biol 13:3202-3212

26. Tan NY, Khachigian LM 2009 Sp1 phosphorylation and its regulation of gene transcription. Mol Cell Biol 29:2483-2488

27. Casey JL, Koeller DM, Ramin VC, Klausner RD, Harford JB 1989 Iron regulation of transferrin receptor mRNA levels requires iron-responsive elements and a rapid turnover determinant in the $3^{\prime}$ untranslated region of the mRNA. EMBO J 8:36933699

28. Seiser C, Posch M, Thompson N, Kuhn LC 1995 Effect of transcription inhibitors on the iron-dependent degradation of transferrin receptor mRNA. J Biol Chem 270:29400-29406

29. Belasco JG 2010 All things must pass: contrasts and commonalities in eukaryotic and bacterial mRNA decay. Nat Rev Mol Cell Biol 11:467-478 